4 Pratter MR. Cough and the common cold: ACCP evidence-based clinical practice guidelines. Chest 2006; 129: Suppl. 1, 72S-74S.

5 Poe RH, Harder RV, Israel RH, et al. Chronic persistent cough. Experience in diagnosis and outcome using an anatomic diagnostic protocol. Chest 1989; 95: 723-728.

Kwon NH, Oh MJ, Min TH, et al. Causes and clinical features of subacute cough. Chest 2006; 129: $1142-1147$.

Waites K, Bébéar C, Robertson JA, et al. Laboratory Diagnosis of Mycoplasma Infections. Cumitech 34. Washington DC, American Society for Microbiology, 2001.

8 Kong F, Gordon S, Gilbert GL. Rapid-cycle PCR for detection and typing of Mycoplasma pneumoniae in clinical specimens. J Clin Microbiol 2000; 38: 4256-4259.

9 Liu YF, Gao Y, Chen MF, et al. Etiological analysis and predictive diagnostic model building of communityacquired pneumonia in adult outpatients in Beijing, China. BMC Infect Dis 2013; 13: 309.

10 Braman SS. Postinfectious cough: ACCP evidence-based clinical practice guidelines. Chest 2006; 129: Suppl. 1, 138S-146S.

\title{
Maternal 25-hydroxyvitamin D levels in relation to offspring respiratory symptoms and infections
}

\section{To the Editor:}

Recently, there has been an increasing interest in the immunomodulatory effects of vitamin D. Several studies have suggested detrimental effects of insufficient 25-hydroxyvitamin D (25(OH)D) levels on the innate and acquired immune system, which may contribute to the development of infections and atopic and allergic conditions [1-4]. Children and pregnant and lactating females have been identified as groups with a high risk of 25(OH)D insufficiency [5]. Low maternal serum 25(OH)D levels in pregnancy may contribute to increased risk of infections and atopic outcomes in offspring. Previous studies demonstrate inconsistency regarding relationships between maternal vitamin D intake, serum 25(OH)D levels and umbilical cord $25(\mathrm{OH}) \mathrm{D}$ levels with these outcomes in offspring [6-10]. We aimed to explore relationships between maternal serum $25(\mathrm{OH}) \mathrm{D}$ levels during late pregnancy and parent-reported respiratory tract symptoms and doctor-diagnosed lower respiratory tract infections (LRTI) in early childhood in a large cohort study.

The study sample consisted of 2025 mother-child pairs from the Southampton Women's Survey with maternal serum 25(OH)D measurement at 34 weeks' gestation (DiaSorin radioimmunoassay; Diasorin, Stillwater, MN, USA) [11]. Follow-up was at children's age 6 months $(\mathrm{n}=2025), 12$ months $(\mathrm{n}=1946)$ and 2 years $(n=1876)$. Parents were asked whether the child had suffered from any of the following since the last visit: one or more episodes of chest wheezing/whistling, waking at night coughing for three or more nights in a row (prolonged cough), one or more episodes of croup or a croupy cough, bouts of vomiting or diarrhoea $\geqslant 2$ days, or a doctor-diagnosed chest infection, bronchitis, bronchiolitis, pneumonia and/or ear infection. Chest infection, bronchitis, bronchiolitis and pneumonia were combined into one variable labelled "LRTI". Binary variables were created for each outcome. Relative risks were calculated using Poisson regression with robust variance with serum $25(\mathrm{OH}) \mathrm{D}>75 \mathrm{nmol} \cdot \mathrm{L}^{-1}$ as the reference category [12]. All analyses were adjusted for child's sex, birthweight and gestational age, and for maternal age at childbirth, educational level, prepregnancy body mass index, parity, ethnicity, smoking in pregnancy and duration of breastfeeding. The study was approved by the Southampton and South West Hampshire Research Ethics Committee (276/97, 307/97, 089/99 and 06/Q1702/104). Consent was obtained before the inclusion of participants.

Median (interquartile range) late-pregnancy serum 25(OH)D level was $59.0(40.6-84.3) \mathrm{nmol} \cdot \mathrm{L}^{-1}$. Lower latepregnancy serum $25(\mathrm{OH}) \mathrm{D}$ levels were not associated with increased risk of parent-reported respiratory symptoms or infections in children aged 6 months, 12 months or 2 years. On the contrary, mothers with serum $25(\mathrm{OH}) \mathrm{D}$ levels $<50 \mathrm{nmol} \cdot \mathrm{L}^{-1}$ reported fewer respiratory symptoms and doctor-diagnosed LRTI in their children aged 0-6 months than those with serum 25(OH)D levels $>75 \mathrm{nmol} \cdot \mathrm{L}^{-1}$ (table 1). Additional adjustment for season of blood sampling (April to September versus October to March) did not alter our findings.

Our results do not support an association between low late-pregnancy serum 25(OH)D levels and increased risk of parent-reported offspring respiratory symptoms and infections in early childhood. The positive associations between serum 25(OH)D levels and self-reported respiratory symptoms and LRTI at 0-6 months may be attributable to residual confounding. Thus, health conscious females have higher serum 
TABLE 1 Relative risks for self-reported respiratory symptoms and infections according to clinical serum 25-hydroxyvitamin D $(25(\mathrm{OH}) \mathrm{D})$ levels

Subjects $\mathrm{n} / \mathrm{N}$

25(OH)D

p-value

$<25 \mathrm{nmol} \cdot \mathrm{L}^{-1} \quad 25-49 \mathrm{nmol} \cdot \mathrm{L}^{-1} \quad 50-74 \mathrm{nmol} \cdot \mathrm{L}^{-1} \quad \geqslant 75 \mathrm{nmol} \cdot \mathrm{L}^{-1}$

\begin{tabular}{lc} 
0-6 months & \\
Subjects & 2025 \\
Wheezing & $525 / 2021$ \\
Prolonged cough & $319 / 2019$ \\
Croupy cough & $79 / 2024$ \\
Diagnosed LRTI & $288 / 2021$ \\
Diagnosed ear & $123 / 2024$ \\
infection & \\
Diarrhoea & $363 / 2024$ \\
Vomiting & $215 / 2024$ \\
6-12 months & \\
Subjects & 1946 \\
Wheezing & $601 / 1946$ \\
Prolonged cough & $450 / 1945$ \\
Croupy cough & $142 / 1946$ \\
Diagnosed LRTI & $368 / 1946$ \\
Diagnosed ear & $386 / 1945$ \\
infection & \\
Diarrhoea & $691 / 1944$ \\
Vomiting & $415 / 1944$ \\
12-24 months & \\
Subjects & 1876 \\
Wheezing & $484 / 1876$ \\
Prolonged cough & $441 / 1875$ \\
Croupy cough & $210 / 1876$ \\
Diagnosed LRTI & $382 / 1875$ \\
Diagnosed ear & $506 / 1875$ \\
infection & \\
Diarrhoea & $671 / 1875$ \\
Vomiting & $467 / 1875$ \\
\hline
\end{tabular}

$\begin{array}{cc}100(4.9) & 666(32.9) \\ 0.64(0.44-0.95) & 0.72(0.61-0.87) \\ 0.33(0.16-0.69) & 0.68(0.53-0.88) \\ 0.13(0.02-1.01) & 0.27(0.14-0.53) \\ 0.45(0.24-0.84) & 0.63(0.49-0.81) \\ 0.83(0.41-1.69) & 0.64(0.40-1.01) \\ & \\ 0.99(0.65-1.50) & 0.93(0.73-1.18) \\ 1.04(0.60-1.81) & 0.86(0.63-1.18)\end{array}$

$94(4.8)$

$1.10(0.80-1.52)$

$1.09(0.73-1.62)$

$1.62(0.86-3.04)$

$1.11(0.72-1.71)$

$1.29(0.87-1.92)$

$0.91(0.67-1.25)$

$1.26(0.85-1.88)$

$95(5.1)$

$0.85(0.58-1.25)$

$0.82(0.52-1.29)$

$0.79(0.42-1.48)$

$0.69(0.41-1.14)$

$1.07(0.77-1.50)$

$1.23(0.95-1.57)$

$1.13(0.79-1.61)$

$628(32.3)$
$1.21(1.03-1.43)$
$1.16(0.95-1.42)$
$0.92(0.62-1.36)$
$1.22(0.97-1.54)$
$1.13(0.90-1.42)$
$0.96(0.83-1.12)$
$1.18(0.96-1.45)$

$601(32.0)$

$1.01(0.84-1.22)$

$1.19(0.97-1.45)$

$0.79(0.58-1.08)$

$0.98(0.79-1.22)$

$0.95(0.78-1.14)$

$1.01(0.87-1.18)$

$1.07(0.88-1.30)$

$572(28.2)$
$0.96(0.81-1.15)$
$0.80(0.62-1.02)$
$0.68(0.41-1.12)$
$0.76(0.58-0.99)$
$0.93(0.61-1.93)$
$1.05(0.83-1.33)$
$0.98(0.71-1.35)$

$552(28.4)$

$1.17(0.98-1.39)$

$1.09(0.89-1.35)$

$0.87(0.58-1.32)$

$1.12(0.87-1.42)$

$1.18(0.93-1.48)$

$1.00(0.86-1.16)$

$0.96(0.76-1.20)$

537 (28.6)

$0.92(0.75-1.12)$

$1.07(0.86-1.33)$

$0.76(0.55-1.05)$

$0.92(0.73-1.16)$

$1.04(0.86-1.25)$

$0.96(0.82-1.13)$

$0.98(0.80-1.20)$
687 (33.9)

Reference $\quad 0.000$

Reference $\quad 0.000$

Reference $\quad 0.000$

Reference $\quad 0.000$

Reference $\quad 0.142$

Reference $\quad 0.484$

Reference $\quad 0.733$

672 (34.5)

Reference

Reference

Reference

Reference

Reference

0.163

0.196

0.773

0.155

0.118

Reference $\quad 0.670$

Reference $\quad 0.046$

643 (34.3)

Reference

Reference

Reference

Reference

Reference

0.991

0.328

0.288

0.929

0.857

Reference

0.680

Reference

Data are presented as $n, n(\%)$ or relative risk (95\% Cl), unless otherwise stated. $p$-values were derived from Poisson regression analyses with the log-transformed $25(\mathrm{OH}) \mathrm{D}$ levels as a continuous variable. All analyses were adjusted for child's sex, birthweight and gestational age, and for maternal age at childbirth, educational level, pre-pregnancy body mass index, parity, ethnicity, smoking in pregnancy and duration of breastfeeding. LRTI: lower respiratory tract infection.

25(OH)D levels and may be more prone to report their children's symptoms and/or have an increased rate of consultation of a doctor at a similar level of symptoms. Other factors which may underlie discrepancies between studies include differences in measurement methods of serum 25(OH)D levels and outcome prevalence, the nature of questions asked and reported behaviour [6-10]. The strengths of this study are its size and population-based nature. Limitations include the absence of objective outcomes regarding respiratory symptoms and infections and, thus, the presence of potential recall bias. In addition, awareness about study end-points may have influenced the medical behaviour of parents. Furthermore, 25(OH)D has a half-life of a few weeks, and we did not measure 25(OH)D levels in early pregnancy or post-natally in the offspring. Thus, mediation through $25(\mathrm{OH}) \mathrm{D}$ at other times in development remains a possibility, and clearly these observational data do not allow causality to be determined. Finally, wheezing may be a symptom of airway inflammation of either allergic or infectious cause and does not discriminate between these aetiologies. Randomised controlled trials are essential to clarify the role of vitamin D in pregnancy in relation to childhood respiratory symptoms and infections.

0 @ERSpublications

Low late-pregnancy 25(OH)D levels not associated with offspring parent-reported respiratory symptoms and infections http://ow.ly/sNzSL

Renate T. de Jongh ${ }^{1}$, Sarah R. Crozier ${ }^{2}$, Stefania D’Angelo², Katharine C. Pike ${ }^{3,4}$, Graham Roberts ${ }^{3,4,5}$, Jane S.A. Lucas ${ }^{3,4}$, Hazel Inskip ${ }^{2}$, Keith M. Godfrey ${ }^{2,4}$, Cyrus Cooper ${ }^{2,4,6}$, Nicholas C. Harvey ${ }^{2,4}$ and the Southampton Women's Survey Study Group ${ }^{7}$ 
${ }^{1}$ Dept of Internal Medicine and Endocrinology, VU University Medical Center, Amsterdam, The Netherlands. ${ }^{2} \mathrm{MRC}$ Lifecourse Epidemiology Unit, University of Southampton, Southampton General Hospital, Southampton, ${ }^{3}$ Clinical and Experimental Sciences Academic Unit, Faculty of Medicine, University of Southampton, Southampton, ${ }^{4}$ NIHR Southampton Respiratory Biomedical Research Unit, University of Southampton and University Hospital Southampton NHS Foundation Trust, Southampton, ${ }^{5}$ Human Development and Health Academic Unit, Faculty of Medicine, University of Southampton, Southampton, and ${ }^{6}$ NIHR Musculoskeletal Biomedical Research Unit, University of Oxford, Nuffield Orthopaedic Centre, Oxford, UK. ${ }^{7}$ A list of the Southampton Women's Survey Study Group members and their affiliations can be found in the Acknowledgements section.

Correspondence: N.C. Harvey, MRC Lifecourse Epidemiology Unit, University of Southampton, Southampton General Hospital, Tremona Road, Southampton, SO16 6YD, UK. E-mail: nch@mrc.soton.ac.uk

Received: July 092013 | Accepted after revision: Nov 182013 | First published online: Dec 122013

Support statement: The work within the Southampton Women's Survey has been funded by the Medical Research Council, University of Southampton, British Heart Foundation, Food Standards Agency (contracts N05049 and N05071), National Institute for Health Research (NIHR) Southampton Biomedical Research Centre, NIHR Southampton Respiratory Biomedical Research Unit and University Hospital Southampton NHS Foundation Trust and University of Southampton Research Policy Committee.

Conflict of interest: Disclosures can be found alongside the online version of this article at www.erj.ersjournals.com

Acknowledgements: Southampton Women's Survey Study Group members are as follows. P. Taylor: Osteoporosis Centre, University Hospital NHS Trust, Southampton General Hospital, Southampton, UK; S.M. Robinson: MRC Lifecourse Epidemiology Unit, University of Southampton, Southampton General Hospital; M. Hanson: Institute of Developmental Sciences, University of Southampton, Southampton General Hospital. The authors thank the children and their families who participated in the Southampton Women's Survey and all the research staff and computing staff.

\title{
References
}

1 Abrams SA, Coss-Bu JA, Tiosano D. Vitamin D: effects on childhood health and disease. Nat Rev Endocrinol 2013; 9: $162-170$.

2 Charan J, Goyal JP, Saxena D, et al. Vitamin D for prevention of respiratory tract infections: a systematic review and meta-analysis. J Pharmacol Pharmacother 2012; 3: 300-303.

Weiss ST, Litonjua AA. Vitamin D in asthma and allergy: what next? Eur Respir J 2011; 38: 1255-1257.

4 Hollams EM, Hart PH, Holt BJ, et al. Vitamin D and atopy and asthma phenotypes in children: a longitudinal cohort study. Eur Respir J 2011; 38: 1320-1327.

5 Mulligan ML, Felton SK, Riek AE, et al. Implications of vitamin D deficiency in pregnancy and lactation. Am J Obstet Gynecol 2010; 202: 429.

6 Christesen HT, Elvander C, Lamont RF, et al. The impact of vitamin D in pregnancy on extraskeletal health in children: a systematic review. Acta Obstet Gynecol Scand 2012; 91: 1368-1380.

7 Miyake Y, Sasaki S, Tanaka K, et al. Dairy food, calcium and vitamin D intake in pregnancy, and wheeze and eczema in infants. Eur Respir J 2010; 35: 1228-1234.

8 Pike KC, Inskip HM, Robinson S, et al. Maternal late-pregnancy serum 25-hydroxyvitamin D in relation to childhood wheeze and atopic outcomes. Thorax 2012; 67: 950-956.

9 Morales E, Romieu I, Guerra S, et al. Maternal vitamin D status in pregnancy and risk of lower respiratory tract infections, wheezing, and asthma in offspring. Epidemiology 2012; 23: 64-71.

10 Bäz N, Dargent-Molina P, Wark JD, et al. Cord serum 25-hydroxyvitamin D and risk of early childhood transient wheezing and atopic dermatitis. J Allergy Clin Immunol 2014; 133: 147-153.

11 Inskip HM, Godfrey KM, Robinson SM, et al. Cohort profile: the Southampton Women's Survey. Int J Epidemiol 2006; 35: 42-48.

12 Barros AJ, Hirakata VN. Alternatives for logistic regression in cross-sectional studies: an empirical comparison of models that directly estimate the prevalence ratio. BMC Med Res Methodol 2003; 3: 21.

\section{A specific DAMP profile identifies susceptibility to smoke-induced airway inflammation}

\author{
To the Editor:
}

Chronic obstructive pulmonary disease (COPD) is a major cause of morbidity and mortality, with a worldwide prevalence of $9-10 \%$ [1]. COPD is associated with chronic, neutrophilic inflammation in the lungs, causing destruction of lung parenchyma (emphysema) and/or remodelling of the airways with mucus 